\title{
Prevalence, Symptoms and Duration of Post Acute Effects in SARS-CoV-2 Positive Children - a Nationwide Cohort Study
}

\section{Luise Borch ( $\sim$ luise.borch@rm.dk)}

Regional hospital West jutland https://orcid.org/0000-0003-1054-0601

\section{Mette Holm}

Pediatrics and Adolescent Medicine, Aarhus University Hospital

\section{Maria Knudsen}

Unit of Clinical Biostatistics, Aalborg University Hospital

\section{Svend Ellermann-Eriksen}

Department of Clinical Microbiology, Aarhus University Hospital

\section{Soeren Hagstroem}

Pediatric department, Aalborg University hospital

\section{Research Article}

Keywords: Sars-CoV-2, children, COVID-19, Long COVID, long-term recovery

Posted Date: September 27th, 2021

DOI: https://doi.org/10.21203/rs.3.rs-839948/v1

License: (c) (1) This work is licensed under a Creative Commons Attribution 4.0 International License. Read Full License 


\section{Abstract}

Background: Most children have a mild course of acute COVID-19, but only a few mainly non-controlled studies with small sample size, have evaluated the long-term recovery from SARS-CoV-2 infection in children ('long COVID').

Methods: We conducted a nationwide cohort study of 37,522 children age 0-17 years with RT-PCR verified SARS-CoV-2 infection and a control group of 78,037 randomly selected children. An electronic questionnaire was sent to both groups of children from March 24th until May 9th 2021.

Results: Long COVID symptoms were reported by $12-51 \%$ of SARS-CoV-2 infected children. Among preschool children, fatigue Risk Difference (RD) 0.05 (Cl 0.04-0.06), loss of smell RD 0.01 (Cl 0.01-0.01), loss of taste RD 0.01 ( $\mathrm{Cl}$ 0.01-0.02) and muscle weakness RD 0.01 ( $\mathrm{Cl} \mathrm{0.00-0.01)} \mathrm{were} \mathrm{statistically} \mathrm{significant}$ symptoms of 'long COVID'.

Among school children the most significant symptoms were loss of smell RD $0.12(\mathrm{Cl} 0.12-0.13)$, loss of taste RD 0.10 ( $\mathrm{Cl}$ 0.09-0.10), fatigue RD 0.05 ( $\mathrm{Cl}$ 0.05-0.06), respiratory problems RD 0.03 ( $\mathrm{Cl} 0.03-0.04)$, dizziness RD 0.02 ( $\mathrm{Cl}$ 0.02-0.03), muscle weakness RD 0.02 (Cl 0.01-0.02), and chest pain RD 0.01 (Cl 0.01-0.01).

Children in the control group experienced significantly more concentration difficulties, headache, muscleand joint pain, cough, nausea, diarrhea and fever than the SARS-CoV-2 infected. In most children 'long COVID' symptoms resolved within 1-5 months.

Conclusions: This study provides new evidence of 'long COVID' symptoms in children.

Trial registration number: The study was approved by The Danish Health Data Authority and registered at Central Denmark region (\# 1-16-02-621-20).

\section{Introduction}

Compared to adults children have a milder course of acute COVID-19 [1, 2]. Moreover, SARS-CoV-2 prevalence is lower in the pediatric population. Hence, pediatric cases constitute $11.8 \%$ (age $0-17$ years), $15.6 \%$ (age 0-18 years) and 10\% (age 0-19 years) in USA, Italy, and Denmark, respectively [3-5]. In Denmark only $0.08 \%$ of children with COVID-19 needed hospitalization[6]. Worldwide, deaths from COVID19 in children also remains rare, at 0.17 per 100.000 population, comprising $0.48 \%$ of the estimated total mortality from all causes in a normal year[7].

As the cumulated incidence of SARS-CoV-2 infection increases, a growing concern arises on persistent multiorgan symptoms after the acute infection, commonly known as 'long COVID'. Long COVID is used to describe signs and symptoms that continue or develop after acute COVID-19, and are not explained by an alternative diagnosis. According to NICE guideline, 'long COVID' includes both on-going symptomatic 
COVID-19 (from 4 to 12 weeks after acute covid-19) and post-COVID-19 syndrome (12 weeks or more after acute covid-19)[8].

There are increasing reports on 'long COVID' in adults[2, 9-12]. However, only a few studies have evaluated the long-term recovery from COVID-19 in children[13-16]. Only one study included a control group[16], and common for all studies is a small sample size. These studies reported that $4-53 \%$ of children experienced post-acute COVID-19 symptoms[13-16] including insomnia, respiratory symptoms, nasal congestion, fatigue, muscle and joint pain, concentration difficulties and loss of smell and taste[13-16]. The lack of pediatric studies, demonstrate a need for further epidemiological data collection in order to quantify and characterize 'long COVID' in non-hospitalized children.

The aim of this study was to document the prevalence, symptoms and duration of 'long COVID' in a nationwide cohort of SARS-CoV-2 infected children $<18$ years. A control group of children who had not been tested positive for SARS-CoV-2 was included for evaluation of symptom relation to SARS-CoV-2 infection.

\section{Materials And Methods}

We conducted a national cohort study of 37,522 children age $0-17$ years with verified SARS-CoV-2 infection by polymerase chain reaction (RT-PCR) and a control group of 78,037 randomly selected children. The study was approved by The Danish Health Data Authority and registered at Central Denmark region (\# 1-16-02-621-20). Ethical approval was not required.

At birth or time of immigration, all Danish citizens are assigned a unique personal identification number registered in the Danish Civil Population Register[17]. This is used in all healthcare contacts, facilitating confidential linkage between registers in Denmark. For the present study, The Danish Health Data Authority linked social security numbers from all Danish children to the national microbiology database. This linkage generated a complete list of all Danish children age $0-17$ years with RT-PCR verified SARSCoV-2 infection at any date between January 27th 2020 and March 19th 2021.

An electronic questionnaire (REDCap) was sent to all SARS-CoV-2 positive children as well as to the control group from March 24th until May 9th 2021. The questionnaire was sent out twice during this period, including a reminder after 7-10 days. Both groups of children had a four-week response time.

The SARS-CoV-2 positive group had the questionnaire sent directly to the parents' secure and private digital post-box (e-boks), which is linked to social security numbers. Adolescents from 15 years received the questionnaire themselves due to legal rights, with advice to fill it out with parental support. The questionnaire was sent out to 6.674 children age $0-5$ years, and 30.848 age $6-17$ years.

For the control group the questionnaire was sent out to parents of children age $0-17$ years, who attended public school or day-care in five municipalities in Denmark (Aalborg, Herning, Aarhus, Randers and Frederiksberg). The parents received the questionnaire directly by the school or day-care's online 
communication platform. A total of 45,240 school children (age 6-17 years) and 32,797 children in daycare (age $0-5$ years) received the questionnaire. In order to exclude children who had been tested positive for SARS-CoV-2 from the control group, previous SARS-CoV-2 infection was addressed in the first question with termination of the rest of the questionnaire if confirmed.

The questionnaire for both groups consisted of identical questions regarding demographic information, a history of chronic illness, medication and symptoms lasting for more than four weeks (S1). For children 9 years and older the WHO-5 well-being index questions were included[18].

\section{Statistical analysis}

Statistical analysis was done using StataMP 17.

Binomial regressions were fitted to estimate risk difference (RD) in percentage point with $95 \%$ confidence interval for each long covid symptom as a function of SARS-CoV-2.

Unpaired two-sample t test with unequal variances was used to determine if there was a significant difference between the means of two groups $(a=0.05)$.

\section{Results}

Of the 37,522 SARS-CoV-2 infected children, a total of 16,836 (44.9\%) responses were returned (44.6\% age $0-5$ years and $39.1 \%$ age $6-17$ years). In the control group, a total of $16,620(21.3 \%)$ responded to the questionnaire ( $21 \%$ age $0-5$ years and $18.3 \%$ age $6-17$ years).

Mean age for children $0-5$ years was 2.7 years (SARS-CoV-2 positive) and 2.8 years (control group), mean difference -0.1 ( $\mathrm{Cl}-0.17--0.02$ ). Mean age for children $6-17$ years was 12.0 years (SARS-CoV-2 positive) and 10.5 years (control group), mean difference 1.5 (Cl 1.41-1.59).

Questionnaires were excluded from analyses in case of missing data regarding symptoms, age or gender of the child. When answering the questionnaire some of the adolescents had turned 18 years since the date of their SARS-CoV-2 positive PCR test, these were excluded from the analysis. Moreover, respondents with less than four weeks from PCR testing, were also excluded from the analysis of 'long COVID'. A total of 15.041 SARS-CoV-2 positive children and 15.080 children in the control group were eligible for inclusion in the study (Fig. 1).

Depending on age, $12-51 \%$ of verified SARS-CoV-2 infected children experienced symptoms four weeks or more after being diagnosed with SARS-CoV-2-infection (Fig. 2). The number of SARS-CoV-2 infected children, who experienced 'long COVID' symptoms increased with increasing age (Fig. 2). The most common 'long COVID' symptoms were fatigue, loss of smell, and loss of taste, headache, and concentration difficulties (Fig. 3). 
In the control group, 15-38\% (depending on age) experienced symptoms lasting more than 4 weeks. In this group of children, the most commonly reported symptoms were concentration difficulties, cough, headache and fatigue (Fig. 3). Five percent of children in both groups suffered from a chronic disease, mainly a respiratory diagnosis ( $51 \%$ of the SARS-CoV-2 children with a chronic disease).

In order to compare the SARS-CoV-2-group to the control group, the children were divided into two age groups: pre-school 0-5 years and school children 6-17 years, and risk differences were estimated (RD). This comparison was made in order to evaluate the impact of confounders such as other infections, school lockdown and social distancing during the COVID-19 pandemic.

Among pre-school children, fatigue RD 0.05 (Cl 0.04-0.06), loss of smell RD 0.01 ( $\mathrm{Cl} 0.01-0.01)$, loss of taste RD 0.01 ( $\mathrm{Cl} 0.01-0.02)$ and muscle weakness RD 0.01 ( $\mathrm{Cl}$ 0.0-0.01) were statistically significant symptoms of 'long COVID' (Fig. 4 and S2). Correspondently, the most significant reported 'long COVID' symptoms in school children were loss of smell RD 0.12(Cl 0.12-0.13), loss of taste RD $0.10(\mathrm{Cl} 0.09$ 0.10 ), and fatigue RD 0.05 ( $\mathrm{Cl} 0.05-0.06$ ). The risk difference was less but still significant in reported respiratory problems RD 0.03 ( $\mathrm{Cl} 0.03-0.04$ ), dizziness $\mathrm{RD} 0.02$ ( $\mathrm{Cl} 0.02-0.03)$, muscle weakness $\mathrm{RD} 0.02$ (Cl 0.01-0.02), and chest pain RD 0.01 (Cl 0.01-0.01) (Fig. 4 and S2).

Seven percent of the previously SARS-CoV-2 infected children, who experienced 'Iong COVID' symptoms, were asymptomatic during the acute infection.

Surprisingly, symptoms with duration of more than 4 weeks were also common in the control group. Children age 0-5 years experienced significantly more cough, fever, concentration difficulties, and diarrhea than children in the SARS-CoV-2 group. Correspondently, 6-17 year old controls were more prone to concentration difficulties, headache, nausea, muscle- and joint pain, cough, diarrhea and fever than their previously SARS-CoV-2 positive peers (Fig. 4 and S2).

Follow-up time from verified SARS-CoV-2 to time of questionnaire completion differed from child to child. Thus an exact duration of symptoms in all participants cannot be reported by this study. However, depending on age, symptoms resolved in a minimum of $54-75 \%$ of children within $1-5$ months (Fig. 5 , panel A). Thirty-nine percent of previously SARS-CoV-2 infected respondents reported having ongoing symptoms. The duration of these ongoing symptoms was addressed by calculation of difference between the known date of verified SARS-CoV-2 infection and the questionnaire response date. Length of follow-up for the children reporting on-going symptoms are illustrated in Fig. 5, panel B.

The well-being of children older than 9 years was evaluated by WHO5 in order to analyze whether symptoms could reflect a low sense of well being, and as such not a direct effect of SARS-CoV-2 infection. Comparing the control group to the whole cohort of SARS-CoV-2 infected children documented, that previously SARS-CoV-2 infected had a higher sense of well-being compared to the control group, WHO-score difference 4 (Cl 3.5-4.8). However, children in both groups, who experienced symptoms, had a worse sense of well being compared to children within the same group but without symptoms, SARS-CoV- 
2 positive: WHO-score difference 12 (Cl 11.1-12.8) control group: WHO-score difference $17.2(\mathrm{Cl} 16.2-$ 18.3).

\section{Discussion}

To our knowledge this is the first study to document prevalence, symptoms and duration of long covid in such a large national cohort study comparing RT-PCR verified SARS-COV-2 infected children to a control group of randomly selected children, who have not previously been tested positive for SARS-CoV-2.

In the present study, $12-51 \%$ of SARS-CoV-2 positive children experienced 'long COVID' symptoms. The age distribution of symptoms differed with older school children being more frequently affected compared to younger school- and preschool children. However, from an identical list of symptoms, 15$38 \%$ of children in the control group also reported symptoms lasting 4 weeks or more. Two previous pediatric studies reported that between $8-66 \%$ of children experience 'long COVID' symptoms $[14,15]$. However, none of these studies included a control group. One pediatric study included a control group, but only had a sample size of 77 children experiencing symptoms lasting for more than 28 days[16], and as such could be categorized as 'long COVID' symptoms according to NICE guideline[8].

Comparing the responses from SARS-CoV-2 infected children to a control group, we found that the most common statistically significant 'long COVID' symptoms were fatigue, loss of smell and loss of taste, and to a lesser extend muscle weakness, chest pain, dizziness and respiratory problems. Loss of smell and taste have previously been reported as symptoms among SARS-CoV-2 positive adolescents [13] and adults[19].

During the COVID-19 pandemic governmental interventions have been introduced in order to reduce transmission of SARS-CoV-2. Quarantine regimes, increased hand hygiene, school and daycare lock down, closure of sports and leisure activities, and social distancing are among the numerous interventions. Reports have raised concern on the negative impact of these social implications on children's mental health[20]. Bearing this in mind it is crucial to compare reported long covid symptoms in previously SARS-CoV-2 positive children to a cohort representative of the background population. Describing 'long COVID' in a pediatric population without a control group could otherwise overestimate 'long COVID' symptoms.

Concentration difficulties, headache, muscle- and joint pain, cough, nausea, diarrhea and fever, have previously been described as 'long COVID' symptoms of SARS-CoV-2 infection in children in noncontrolled trials $[14,15,21]$. However, we found that these symptoms were statistically more significant in the control group. Our study also documented that children in the control group had a lower WHO-5score compared to SARS-CoV-2 positive children. Therefore, it should be considered whether concentration difficulties, headache, muscle- and joint pain as well as nausea, could be symptoms reflecting the negative impact of the social implications of the pandemic on children's mental and physical health. The reason for reports of more frequent fever occurrence in a randomly selected control group can only be speculated, since questions on specific method of temperature evaluation or accompanying symptoms 
were not included. In general, the first year of the pandemic have had a remarkably low incidence of otherwise high incidence infections, such as respiratory syncytial virus and influenza virus[22, 23]. We would therefore have expected a low occurrence of fever.

The duration of 'long COVID' symptoms is an important issue to address with implications for the children and families. In our pediatric cohort, most children recovered within a maximum of 1-5 months. Recovery time of 2 weeks-3 months have previously been described in non-controlled pediatric studies[14, 15].

We aimed to document whether 'long COVID' symptoms could reflect isolation, corona restrictions and lock downs. Our analysis showed that children presenting with 'long COVID' symptoms reported a higher sense of well being compared to children with symptoms who have never been tested positive for SARSCoV-2. As such, fatigue, loss of smell and loss of taste, muscle weakness, chest pain, dizziness ad respiratory problems cannot be assigned to psychological sequelae of social restrictions but must be recognized as 'long COVID' symptoms.

There are significant limitations to this observational study. Firstly, our questionnaire was not validated in a larger pilot study. Moreover, data relies on participants' retrospectively parent- or self-reported symptoms with a risk of recall bias. However, SARS-CoV-2 is an infection with increased focus in society. As such, participants have possibly been extremely aware of symptoms during their infection, and therefore recall bias is assumed to be minimal. Secondly, the control group might include children who have had SARS-CoV-2 infection without having undergone testing. This is a risk since children have no or only few symptoms of acute COVID-19. Moreover, parents might be more reluctant to let children undergo testing due to the invasive and unpleasant procedure. Children in the control group have never been tested positive for SARS-CoV-2. Nevertheless, seroprevalence data suggest an infection rate of 2-3 times higher than corresponding PCR results in the pediatric population[24]. The potential presence of children with previous SARS-CoV-2 infection in the control group would underestimate the symptoms of 'long COVID'. Thirdly, it could be speculated that children in the control group and the SARS-CoV-2 group who experience symptoms are more eager to respond to the questionnaire than asymptomatic children. This could lead to selection bias and result in an overestimation of reported symptoms. However, the study may also suffer from non-response bias where non-symptomatic controls may be under-represented. The number of children answering the questionnaire was higher among SARS-CoV-2 positive children than in the control group ( $44.9 \%$ versus $21.3 \%$ ). The higher response rate in the group of children with previous SARS-CoV-2 infection could be expected since these children and families might have higher awareness on potential 'long COVID' symptoms, and a potential increased desire for knowledge about long covid. However, in both groups, the children who answered the questionnaire were an equal age distribution of the children who received the questionnaire.

The strengths of this study are primarily the large sample size and inclusion of a control group. Additionally, the Danish Health Data Authority has a nationwide coverage, and all Danish citizens have universal tax-funded health insurance. Therefore our study population did not rely on access to health- 
care services, and RT-PCR tests for SARS-CoV-2 are free of charge in Denmark minimizing selection-bias. The infection status of all SARS-CoV-2 positive children has been established by nationally validated RTPCR, eliminating major misclassification of infection status. Moreover, the results of the SARS-CoV-2 test are linked to the unique personal identification number registered in the Danish Civil Population Register[17] and the national microbiology database making it a complete cohort of SARS-CoV-2 infected children below 18 years from the start of the pandemic in Denmark until 19th of March 2021.

The control group consists of randomly selected children age $0-17$ years from five municipalities that include both larger cities as well as rural areas. As such, the control group is considered to represent the general Danish population of children. The strengths mentioned above increase the generalizability of our results. However, it is important to consider that an observational study based on a questionnaire with subjective responses and without objective examination of the children, can only provide us with one piece of the puzzle. Further studies are needed to increase knowledge of 'long COVID' in the pediatric population.

In conclusion, to date this study is the largest study on post acute effects in SARS-CoV-2 positive children also including a control group. It provides new evidence that symptoms of 'long COVID' in children are fatigue, loss of smell and loss of taste, dizziness, muscle weakness, chest pain and respiratory problems.

These symptoms cannot be assigned to psychological sequelae of social restrictions. Symptoms such as concentration difficulties, headache, muscle- and joint pain as well as nausea may be related to other factors than SARS-CoV-2 infection. In most cases 'long COVID' symptoms resolve within 1-5 months.

\section{Declarations}

\section{Transparency statement}

The lead author affirms that this manuscript is an honest, accurate, and transparent account of the study being reported. No important aspects of the study have been omitted. The study has been performed as originally planned.

\section{Funding}

The foundation of 1844 . The foundation had no role in in study design, collection of data, analysis and interpretation of data, writing of the report or in the decision to submit the paper for publication.

\section{Declarations}

All authors certify that they have no affiliations with or involvement in any organization or entity with any financial interest or non-financial interest in the subject matter or materials discussed in this manuscript.

\section{References}


1. Swann OV, Holden KA, Turtle L et al (2020) Clinical characteristics of children and young people admitted to hospital with covid-19 in United Kingdom: prospective multicentre observational cohort study. BMJ 370:m3249. https://doi.org/10.1136/bmj.m3249

2. Petersen MS, Kristiansen MF, Hanusson KD et al (2020) Long COVID in the Faroe Islands - a longitudinal study among non-hospitalized patients. Clin Infect Dis Off Publ Infect Dis Soc Am. https://doi.org/10.1093/cid/ciaa1792

3. Ugentlige opgørelser med overvågningsdata. https://covid19.ssi.dk/overvagningsdata/ugentligeopgorelser-med-overvaagningsdata. Accessed 10 Apr 2021

4. EpiCentro COVID-19 integrated surveillance data in Italy. https://www.epicentro.iss.it/en/coronavirus/sars-cov-2-dashboard. Accessed 10 Apr 2021

5. CDC (2020) COVID Data Tracker. In: Cent. Dis. Control Prev. https://covid.cdc.gov/covid-data-tracker. Accessed 10 Apr 2021

6. Hartling UB, Holm M, Glenthoej JP et al (2021) The Need for Hospitalization due to SARS-CoV-2 in Children: A Population-based Estimate. Pediatr Infect Dis J 40:e250-e251. https://doi.org/10.1097/INF.0000000000003127

7. Bhopal SS, Bagaria J, Olabi B, Bhopal R (2021) Children and young people remain at low risk of COVID-19 mortality. Lancet Child Adolesc Health 0: https://doi.org/10.1016/S2352-4642(21)00066-3

8. (2020) COVID-19 rapid guideline: managing the long-term effects of COVID-19. National Institute for Health and Care Excellence (UK), London

9. Townsend L, Dyer AH, Jones $\mathrm{K}$ et al (2020) Persistent fatigue following SARS-CoV-2 infection is common and independent of severity of initial infection. PloS One 15:e0240784. https://doi.org/10.1371/journal.pone.0240784

10. Carvalho-Schneider C, Laurent E, Lemaignen A et al (2021) Follow-up of adults with noncritical COVID-19 two months after symptom onset. Clin Microbiol Infect 27:258-263. https://doi.org/10.1016/j.cmi.2020.09.052

11. Doykov I, Hällqvist J, Gilmour KC et al (2020) "The long tail of Covid-19" - The detection of a prolonged inflammatory response after a SARS-CoV-2 infection in asymptomatic and mildly affected patients. F1000Research 9:1349. https://doi.org/10.12688/f1000research.27287.2

12. Lund LC, Hallas J, Nielsen $\mathrm{H}$ et al (2021) Post-acute effects of SARS-CoV-2 infection in individuals not requiring hospital admission: a Danish population-based cohort study. Lancet Infect Dis. https://doi.org/10.1016/S1473-3099(21)00211-5

13. Kumar L, Kahlon N, Jain A et al (2021) Loss of smell and taste in COVID-19 infection in adolescents. Int J Pediatr Otorhinolaryngol 142:110626. https://doi.org/10.1016/j.ijporl.2021.110626

14. Say D, Crawford N, McNab S et al (2021) Post-acute COVID-19 outcomes in children with mild and asymptomatic disease. Lancet Child Adolesc Health 5:e22-e23. https://doi.org/10.1016/S23524642(21)00124-3

15. Buonsenso $D$, Munblit $D$, Rose $C D$ et al Preliminary evidence on long COVID in children. Acta Paediatr n/a: https://doi.org/10.1111/apa.15870 
16. Molteni $\mathrm{E}$, Sudre $\mathrm{CH}$, Canas LS et al (2021) Illness duration and symptom profile in symptomatic UK school-aged children tested for SARS-CoV-2. Lancet Child Adolesc Health 0:

https://doi.org/10.1016/S2352-4642(21)00198-X

17. Pedersen CB (2011) The Danish Civil Registration System. Scand J Public Health 39:22-25. https://doi.org/10.1177/1403494810387965

18. Allgaier A-K, Pietsch K, Frühe B et al (2012) Depression in pediatric care: is the WHO-Five Well-Being Index a valid screening instrument for children and adolescents? Gen Hosp Psychiatry 34:234-241. https://doi.org/10.1016/j.genhosppsych.2012.01.007

19. D'Ascanio L, Pandolfini M, Cingolani C et al (2021) Olfactory Dysfunction in COVID-19 Patients: Prevalence and Prognosis for Recovering Sense of Smell. Otolaryngol-Head Neck Surg Off J Am Acad Otolaryngol-Head Neck Surg 164:82-86. https://doi.org/10.1177/0194599820943530

20. Loades ME, Chatburn E, Higson-Sweeney N et al (2020) Rapid Systematic Review: The Impact of Social Isolation and Loneliness on the Mental Health of Children and Adolescents in the Context of COVID-19. J Am Acad Child Adolesc Psychiatry 59:1218-1239.e3.

https://doi.org/10.1016/j.jaac.2020.05.009

21. Ludvigsson JF (2021) Case report and systematic review suggest that children may experience similar long-term effects to adults after clinical COVID-19. Acta Paediatr Oslo Nor 1992 110:914921. https://doi.org/10.1111/apa.15673

22. Yeoh DK, Foley DA, Minney-Smith CA et al (2020) The impact of COVID-19 public health measures on detections of influenza and respiratory syncytial virus in children during the 2020 Australian winter. Clin Infect Dis Off Publ Infect Dis Soc Am. https://doi.org/10.1093/cid/ciaa1475

23. Huang QS, Wood T, Jelley L et al (2021) Impact of the COVID-19 nonpharmaceutical interventions on influenza and other respiratory viral infections in New Zealand. Nat Commun 12:. https://doi.org/10.1038/s41467-021-21157-9

24. Stringhini S, Wisniak A, Piumatti G et al (2020) Seroprevalence of anti-SARS-CoV-2 IgG antibodies in Geneva, Switzerland (SEROCoV-POP): a population-based study. Lancet Lond Engl 396:313-319. https://doi.org/10.1016/S0140-6736(20)31304-0

\section{Figures}




\section{CONSORT Flow chart of study participants}
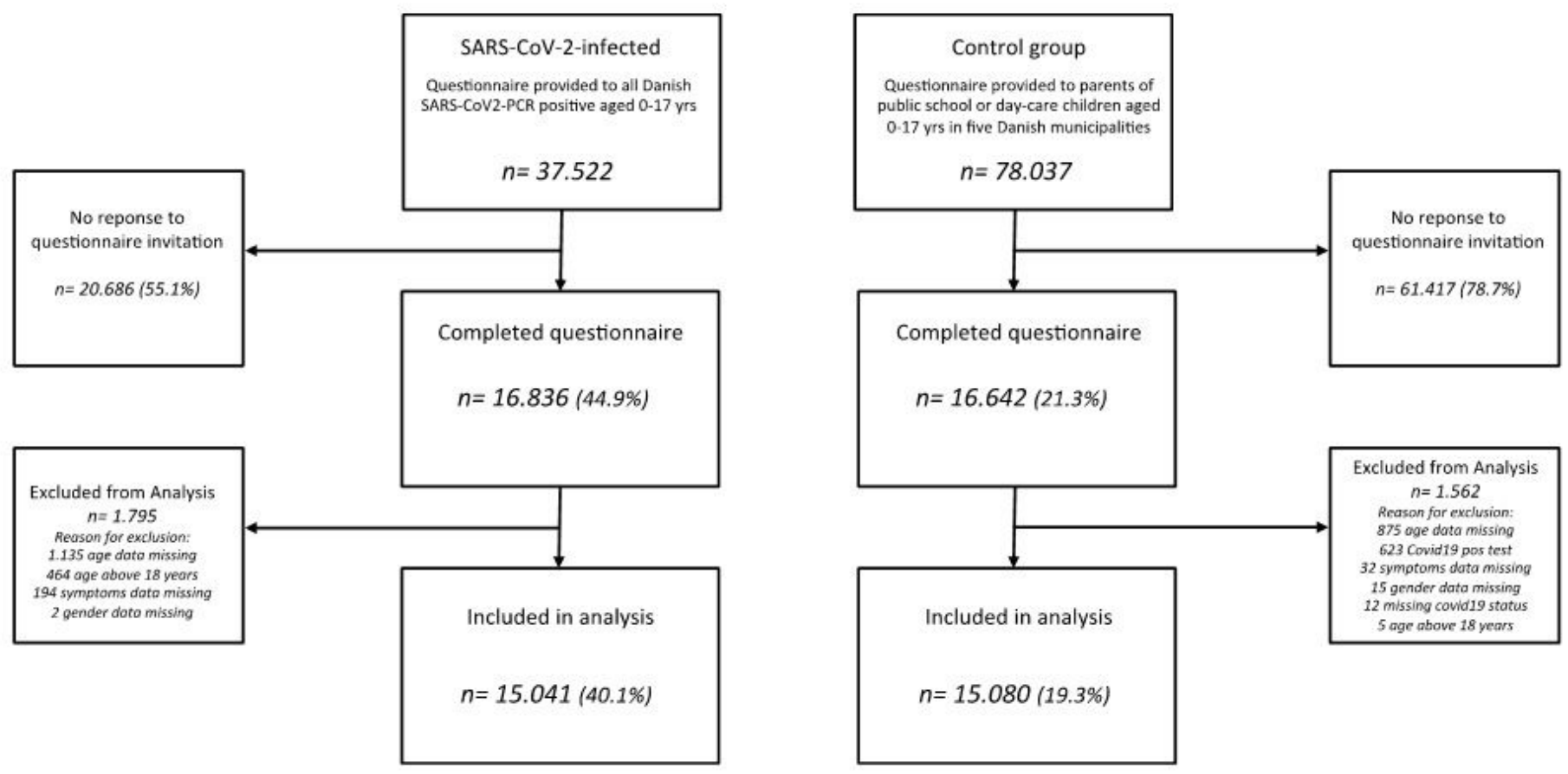

Figure 1

CONSORT Flow chart of study participants Permission to use this image was given by co-author Maria Knudsen, who created the figure. 

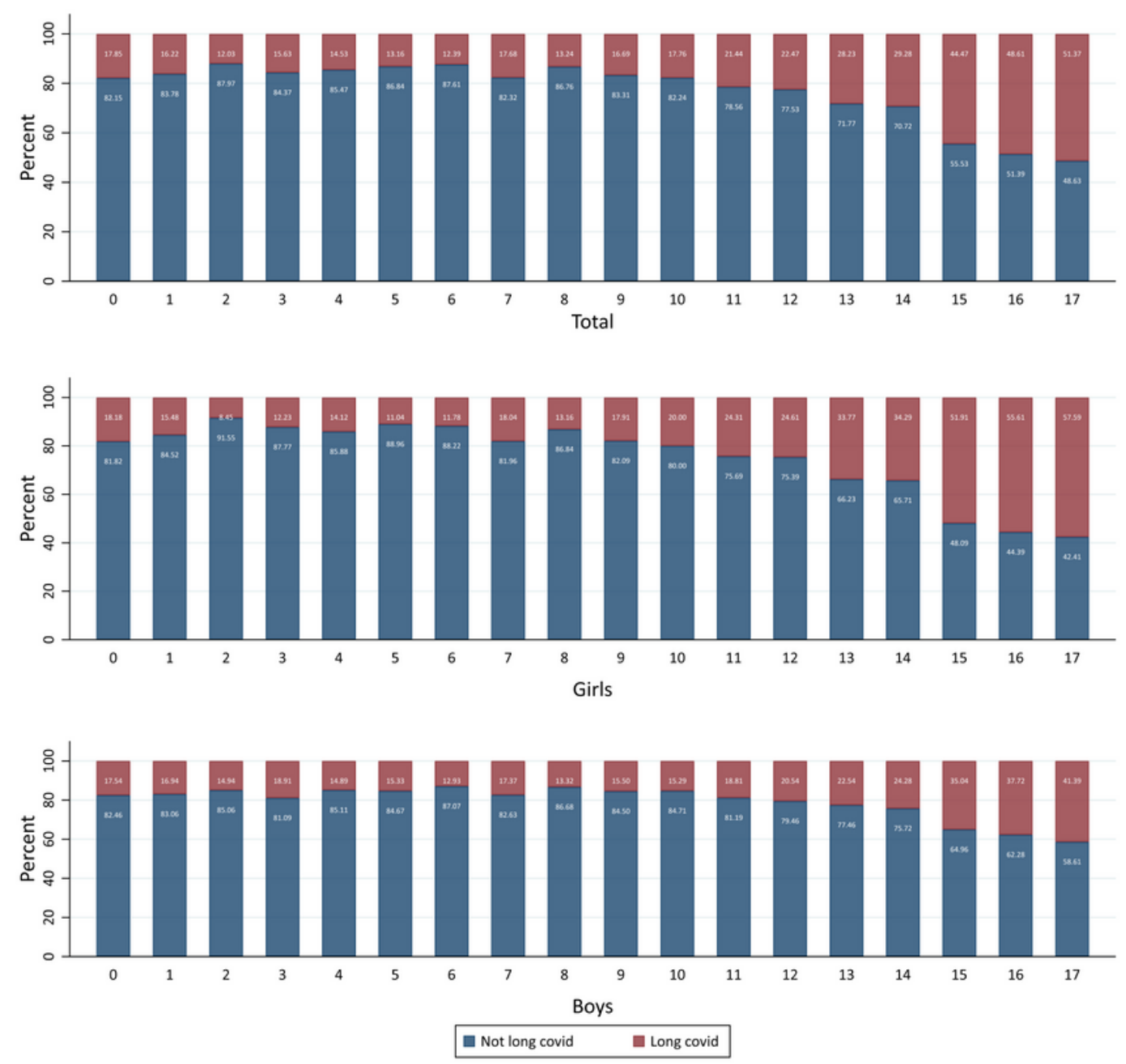

Figure 2

Prevalence of long COVID symptoms. Percentage of SARS-CoV-2 infected children reporting at least one long COVID symptom (red bars) or reporting no symptoms (blue bars). Data are presented as total population of SARS-CoV-2 infected children (upper panel) and by gender (Girls -middle panel, Boys -lower panel). Permission to use this image was given by co-author Maria Knudsen, who created the figure. 
SARS-CoV2 positive

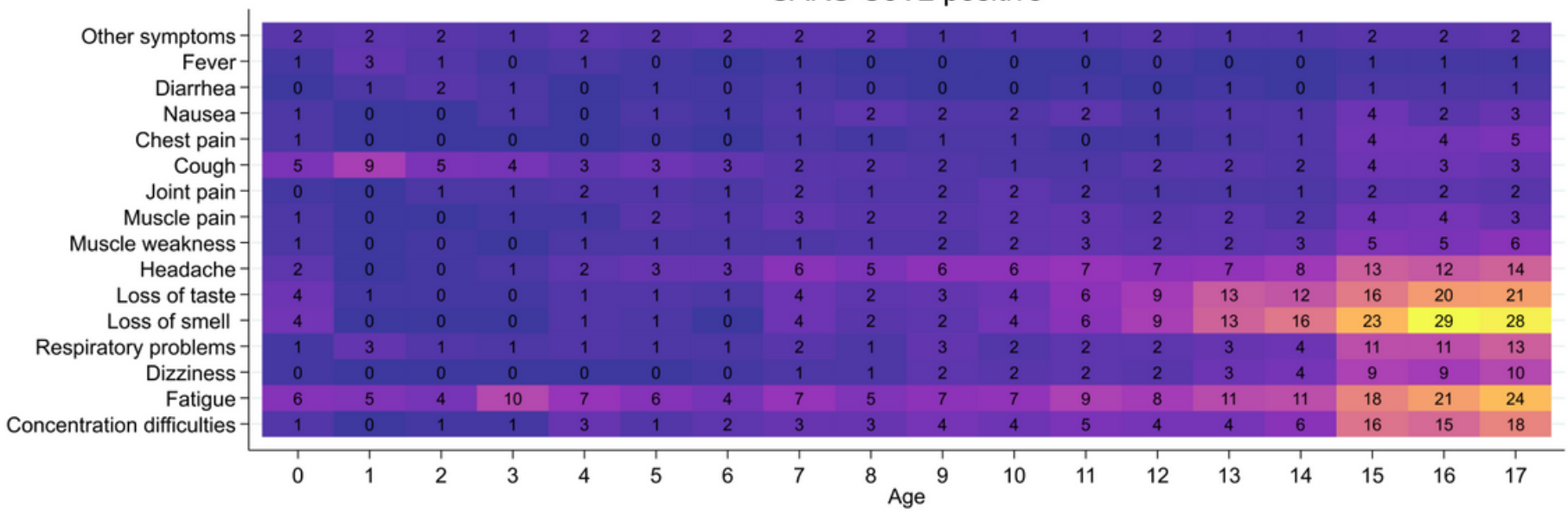

Control group

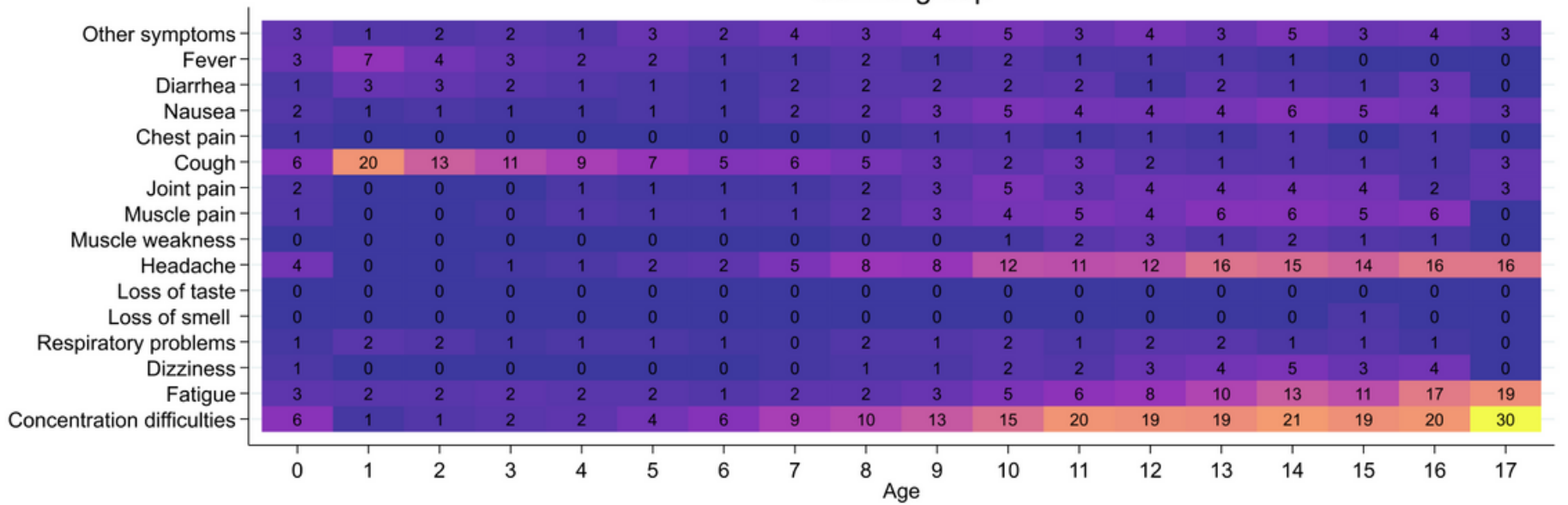

\section{Figure 3}

Heatmap illustrating reported symptoms lasting for more than 4 weeks by SARS-CoV-2 infected children (upper panel) and controls (lower panel). The numbers represent percentage of children reporting the given symptom by one-year age groups. Permission to use this image was given by co-author Maria Knudsen, who created the figure. 
Panel A

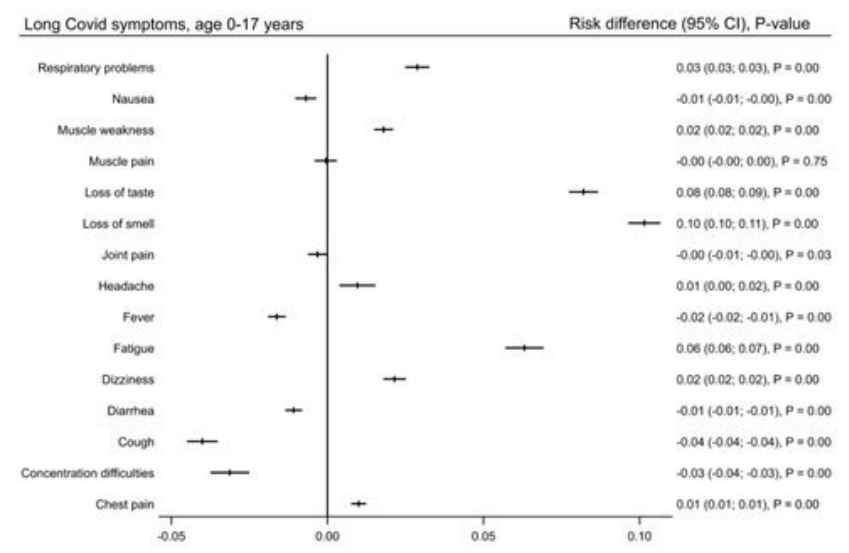

Panel B

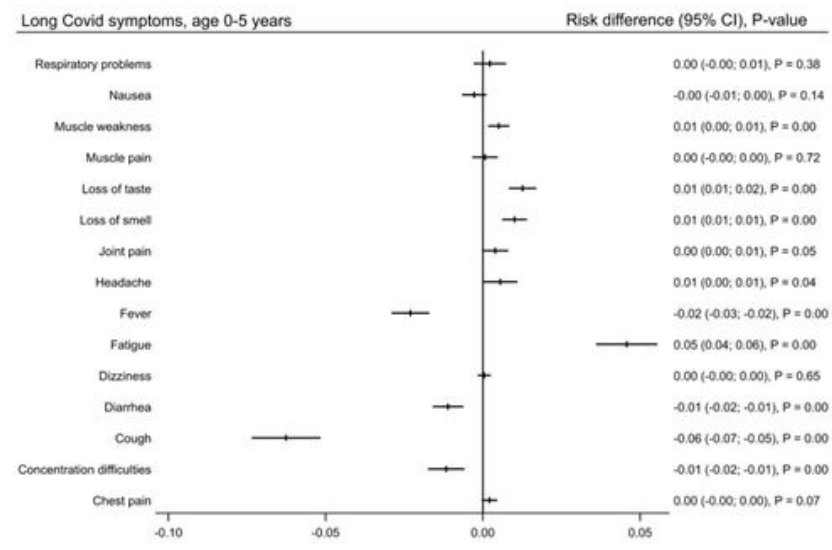

Panel C

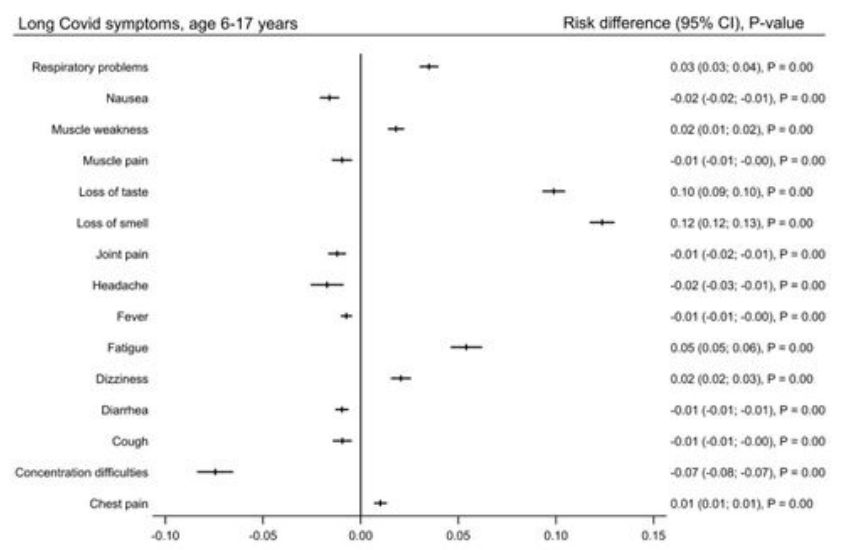

Figure 4

Comparison of symptom prevalence in SARS-CoV-2 infected children and the control group indicated by risk differences (RD) with 95\% confidence interval and p-values. Panel A: comparison of children 0-17 years. Panel B: Sub-group comparison of pre-school children, 0-5 years. Panel C: Sub-group comparison of School children, 6-17 years. Permission to use this image was given by co-author Maria Knudsen, who created the figure. 

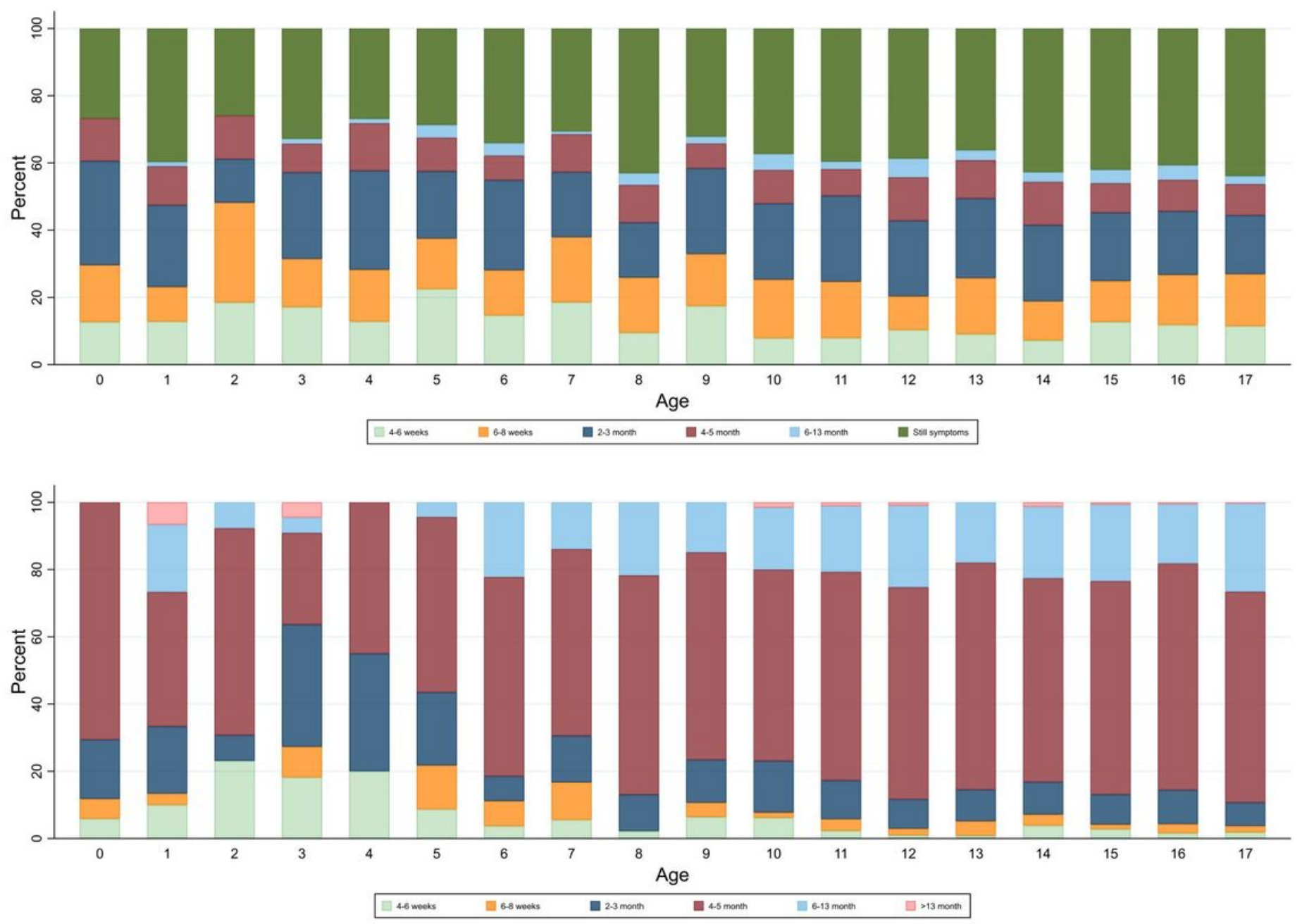

Figure 5

Duration of symptoms from date of positive RT-PCR SARS-CoV-2 test until date for questionnaire completion by one-year age groups. In the upper panel the dark green bars represent percentage of children who reported that their symptoms had not resolved at time of questionnaire completion. The follow-up time of these children's on-ongoing symptoms are illustrated in the lower panel. Permission to use this image was given by co-author Maria Knudsen, who created the figure.

\section{Supplementary Files}

This is a list of supplementary files associated with this preprint. Click to download.

- supplementarymaterial.pdf 